\title{
Impact of Acute Dietary Manipulations on Dual-Energy X-ray Absorptiometry Estimates of Visceral Adipose Tissue
}

Jeffrey S. Forsse ${ }^{1}$, Grant M. Tinsley ${ }^{2}$, Flor E. Morales Marroquín ${ }^{1}$, and Peter W. Grandjean, FACSM 1

${ }^{1}$ Department of Health, Human Performance, and Recreation, Baylor University, Waco, TX., ${ }^{2}$ The Department of Kinesiology and Sport Management, Texas Tech University, Lubbock, TX
Category: Doctoral
Advisor/Mentor: Grandjean, Peter (Peter_Grandjean@baylor.edu)
ABSTRACT

Dual-energy $\mathrm{x}$-ray absorptiometry (DXA) is viewed as a superior method of body composition assessment, but whole-body DXA scans are impacted by variation in pre-assessment activities, such as eating and drinking. DXA software now allows for estimation of visceral adipose tissue (VAT), which has been implicated in a number of diseases. It is unknown to what extent food and fluid intake affect VAT estimates. Purpose: To determine the effects of acute high-carbohydrate $(\mathrm{HC})$ and very low-carbohydrate (VLC) diets on DXA estimates of VAT. Methods: Male and female adults completed two one-day dietary conditions in random order: a VLC $\operatorname{diet}(1-1.5 \mathrm{~g} \mathrm{CHO} / \mathrm{kg})$ and a $\mathrm{HC} \operatorname{diet}(9 \mathrm{~g} \mathrm{CHO} / \mathrm{kg})$. The diets were isocaloric to each other, and all food items were provided to participants. DXA scans were conducted in the morning after an overnight fast and in the afternoon soon after the third standardized meal. VAT volume, mass, and area were obtained, and paired samples t-tests were performed to compare the changes in VAT measures between diets. Results: Fifteen males (age $22 \pm 3, \mathrm{BF} \% 21 \pm 5 \%$ ) and eighteen females (age $21 \pm 2, \mathrm{BF} \% 31 \pm 5 \%$ ) were included in the analysis. The change in VAT volume between the fasted and fed visits was different between diets (HC: $+1.6 \%$; VLC: $-9.2 \%, \mathrm{p}=0.047$ ). There were also trends for differences in VAT mass $(p=0.089)$ and area $(p=0.096)$ changes between diets. Conclusions: Within a single day, VAT estimates are differentially affected by isocaloric HC and VLC diets, with VLC consumption leading to reductions in VAT estimates. The content of the diet on the day of a DXA scan can affect estimates of VAT, which could spuriously influence the categorization of an individual's health risk by DXA VAT estimates. Standardization of food intake prior to scans, preferably in the form of an overnight fast, should be employed to eliminate this important source of error.

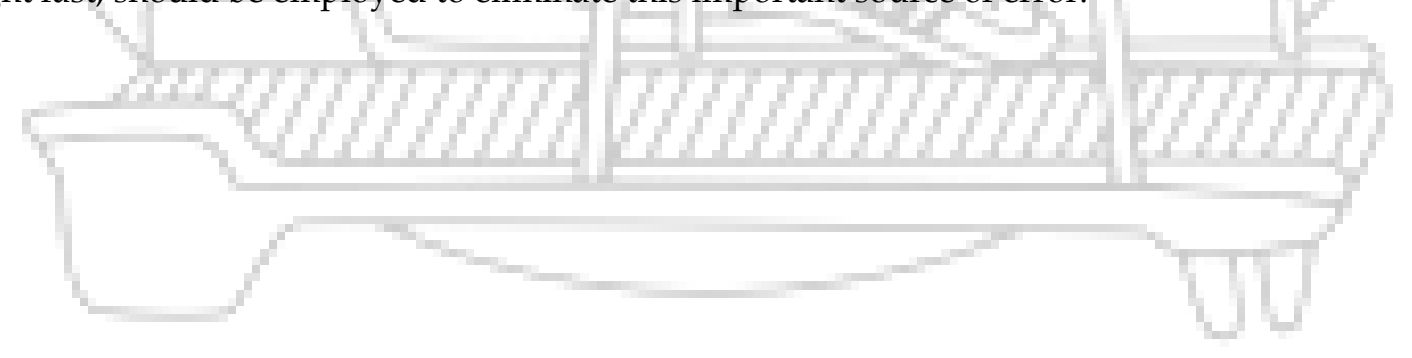

